\title{
ANALYSIS OF FLUID-STRUCTURE INTERACTIONS. A DIRECT SYMMETRIC COUPLED FORMULATION BASED ON THE FLUID VELOCITY POTENTIAL
}

\author{
Lorraine G. Olson $\dagger$ and Klaus-Jürgen Bathe $\ddagger$ \\ Department of Mechanical Engineering, Massachusetts Institute of Technology, Cambridge, MA \\ 02139, U.S.A.
}

\begin{abstract}
We present a symmetric finite element method for solving fluid-structure interaction problems. The formulation uses velocity potentials and a hydrostatic pressure as unknowns in each fluid region, and displacements as unknowns in the solid. The hydrostatic pressure is an unknown variable at only one node per fluid region. A C matrix (multiplied by time derivatives of the nodal variables, but not a damping matrix) enforces the coupling between the variables. The resulting matrix equations are banded and symmetric, making them easy to incorporate in standard displacement-based finite element codes. Several test cases indicate that this approach works well for static, transient, and frequency analyses.
\end{abstract}

\section{INTRODUCTION}

Fluid-structure interactions significantly affect the response of nuclear reactor components, marine platforms, ships, turbines, dams, and piping systems. As a result, much effort has gone into the development of general finite element methods for fluid-structure systems. We restrict ourselves here to fluids modeled as inviscid and irrotational, with no gravity effects. These fluids are assumed to exist in bounded domains and undergo only small motions.

Previously[1], we attempted to solve four categories of fluid-structure interaction problems using displacements as variables in the fluid and the solid (see Fig. 1). We encountered fundamental difficulties modeling solids vibrating in nearly incompressible fluids with this formulation. (See Ref. [1] for a full discussion).

In this paper, we use velocity potentials $(\phi)$ as the nodal variables in the fluid, and introduce a hydrostatic pressure variable $\left(P_{0}\right)$ measured at only one node in each fluid region. Displacements $(U)$ remain the nodal variables in the solid. A $\mathbf{C}$ matrix (not damping) couples the fluid to the solid, and links the pressure to the velocity potential in a fluid region. We will show that this $\phi-U-P_{0}$ method works well for each class of fluid-structure interaction problems attempted in our first paper, including solids vibrating in nearly incompressible fluids. In addition, the $\phi-U-P_{0}$ method offers symmetry, easy incorporation into displacement-based finite clement programs, and reduced fluid degrees of freedom (compared with the displacement method).

Everstine introduced the $\phi-U$ portion of the formulation for transient wave propagation problems[2-5]. Static problems cannot be solved with the $\phi-U$ model alone.

\section{$\dagger$ Graduate student}

$\ddagger$ Professor of Mechanical Engineering.
Most finite element analysts use one of two approaches for these types of fluid-structure interaction problems. Zienkiewicz and Newton[6] use pressure as the unknown in the fluid and displacements in the solid, which yields unsymmetric matrices. Other researchers, including Daniel[ 7,8$]$, Petyt and Lim[9], Muller[10], and Craggs[11] use similar formulations. Many workers have used displacements as the nodal variable in both the solid and the fluid. Among these are Belytschko [e.g. Refs. 12-14], Wilson[15, 16], Bathe and Hahn[17], and Hamdi, Ousset, and Verchery[18]. In a different approach, Morand and Ohayon[19] have investigated mixed symmetric variational formulations. Zienkiewicz and Bettess[20] and Belytschko[21] survey work in this field.

\section{FINITE ELEMENT FORMULATION}

2.1 Virtual work statement of problem

Figure 2 shows the general system under consideration. The solid extends throughout the region $S$, and region $F$ contains the fluid. Surface $I$ defines the fluid-structure interface. On boundary $B$ all displacements and velocities are zero.

In the solid, the variational indicator is[22]

$$
\begin{aligned}
\Pi=\int\left[\frac{1}{2} \int \varepsilon^{T} \mathbf{C}_{s} \varepsilon \mathrm{d} S\right. & -\frac{1}{2} \int \rho \dot{\mathbf{u}}^{T} \dot{\mathbf{u}} \mathrm{d} S \\
& \left.-\int \mathbf{u}^{I T} \mathbf{f}^{I} \mathrm{~d} I\right] \mathrm{d} t,
\end{aligned}
$$

where $\varepsilon=$ strain tensor, $\mathbf{C}_{s}=$ material stressstrain matrix, $\rho=$ density of solid, $\mathbf{u}=$ displacement vector, $\mathbf{f}^{I}=$ surface (interface) force vector, and $t=$ time. Setting variations on $\Pi$ to zero gives the principle of virtual displacements

$$
\int \overline{\boldsymbol{\varepsilon}}^{T} \mathbf{C}_{s} \mathbf{\varepsilon} \mathrm{d} S+\int \rho \overline{\mathbf{u}}^{T} \ddot{\mathbf{u}} \mathrm{d} S=\int \overline{\mathbf{u}}^{I T} \mathbf{f}^{I} \mathrm{~d} I,
$$

where the bar over a variable denotes "virtual". 


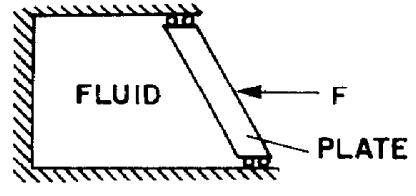

(a)

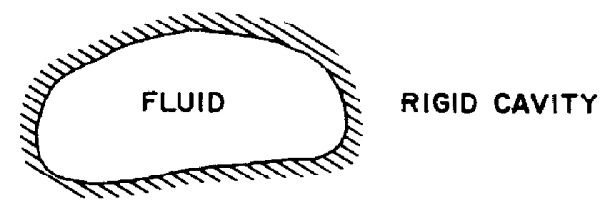

(b)

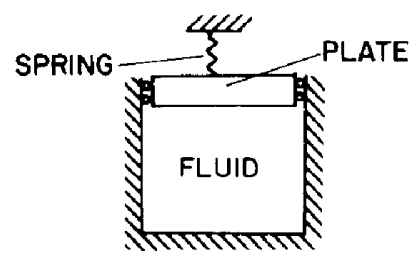

(c)

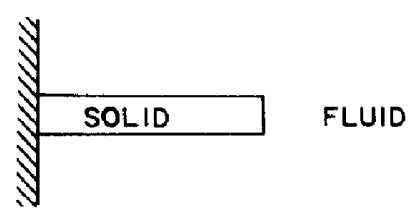

(d)

Fig. 1. Four categories of fluid-structure interaction problems. (a) Category 1: Static problems. (b) Category 2: Natural frequencies of fluid in rigid cavities. (c) Category 3: Fluid vibrating in flexible boundaries. (d) Category 4: Structures vibrating in fluids.

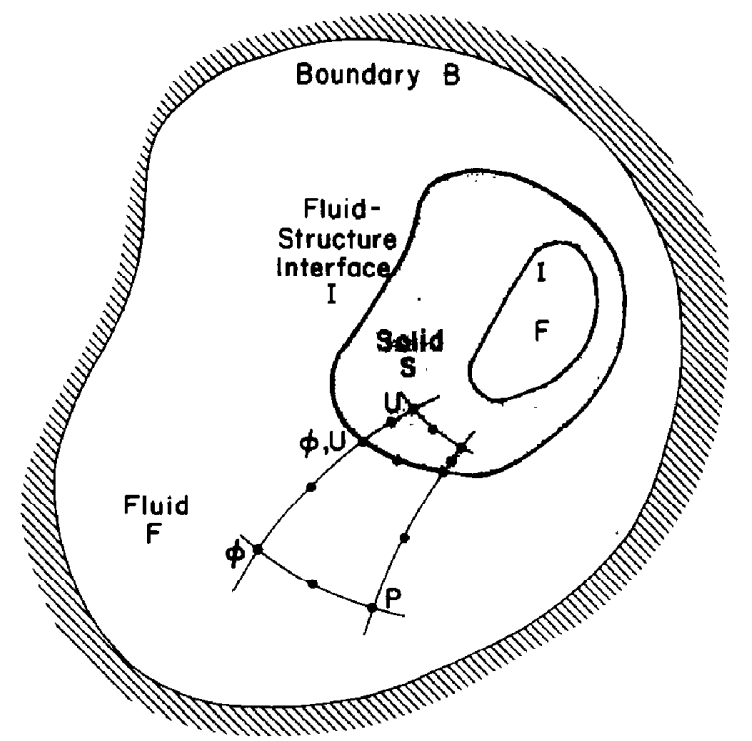

Fig. 2. Fluid-structure problem geometry.
We can separate surface forces into tluid-caused $\left(f^{F}\right)$ and additionally applied forces $\left(f^{E}\right)$ to give

$$
\begin{aligned}
\int \overline{\boldsymbol{\varepsilon}}^{I} \mathbf{C}_{\mathrm{s}} \boldsymbol{\varepsilon} \mathrm{d} S+ & \int \rho \overline{\mathbf{u}}^{I} \ddot{\mathbf{u}} \mathrm{d} S \\
& =\int \overline{\mathbf{u}}^{I I} \mathbf{f}^{L} \mathrm{~d} I+\int \overline{\mathbf{u}}^{I /} \mathbf{f}^{\digamma^{\prime}} \mathrm{d} I
\end{aligned}
$$

For the compressible, inviscid fluid which experiences only small displacements[23], the variational indicator is

$$
\begin{aligned}
& \mathrm{Il}=\int\left[\frac{1}{2} \int \frac{1}{\beta}\left(P_{0}-\rho_{F} \dot{\phi}\right)^{2} \mathrm{~d} F\right. \\
& \frac{-\frac{1}{2} \int \rho_{F} \nabla \phi \cdot \nabla \phi d F}{I I} \\
& \underbrace{\left.-\int\left(P_{0}-\rho_{F} \dot{\phi}^{\prime}\right) u_{N} \mathrm{~d} I\right]}_{\text {III }}] \mathrm{d} t,
\end{aligned}
$$

where $\rho_{F}=$ fluid density, $\phi=$ velocity potential in the fluid, $\beta=$ bulk modulus of fluid, $P_{0}=$ hydrostatic pressure in the fluid, and $\|_{N}=$ externally imposed displacement, normal to the fluid boundary and positive acting into the fluid. Notice that the pressure at any point in the fluid is the sum of the hydrostatic pressure and the pressure due to velocity potentials $\left(-\rho_{F} \bar{\phi}\right)$. As a result, term I represents the compression energy in the fluid volume. Term II is the kinetic energy due to fluid motion. Term III contains the "potential of the external loads", in this case the integral over area of pressure times displacement.

Invoking the stationarity of 11 with respect to $\phi$ and $P_{0}\left(\right.$ not $\left.u_{N}\right)$ gives the "principle of virtual potentials" for the fluid

$$
\begin{aligned}
& \int \frac{1}{\beta} \bar{P}_{0} P_{0} \mathrm{~d} F-\int \frac{\rho_{F}}{\beta} \bar{P}_{0} \dot{\phi} \mathrm{d} F+\int \frac{\rho_{F}}{\beta} \bar{\phi}_{P_{0}} \mathrm{~d} F \\
& \quad-\int \frac{\rho_{F}^{2}}{\beta} \bar{\phi} \ddot{\phi} \mathrm{d} F-\int \rho_{F} \nabla \bar{\phi} \cdot \nabla \phi \mathrm{d} F=\int \bar{P}_{0} u_{N} \mathrm{~d} I \\
& \quad+\int \rho_{F} \dot{\phi}^{\prime} \dot{u}_{N} \mathrm{~d} I .
\end{aligned}
$$

We couple the two virtual work expressions at the common interface $I$. An inviscid fluid exerts only pressure forces on the solid

$$
\mathbf{f}^{F}=-\mathbf{n}\left(P_{0}-\rho_{F} \dot{\phi}^{I}\right)
$$

where

$$
\mathbf{n}^{T}=\left[\begin{array}{lll}
n_{x} & n_{v} & n_{-}
\end{array}\right]
$$

$=$ unit outward normal from solid. 
Equation (3) becomes

$$
\begin{aligned}
\int \overline{\boldsymbol{\varepsilon}}^{T} \mathbf{C}_{s} \varepsilon \mathrm{d} S+\int \rho \overline{\mathbf{u}}^{T} \ddot{\mathbf{u}} \mathrm{d} S=\int \overline{\mathbf{u}}^{I T} \mathbf{f}^{E} \mathrm{~d} I \\
-\int \overline{\mathbf{u}}^{I T} \mathbf{n} P_{0} \mathrm{~d} I+\int \rho_{F} \overline{\mathbf{u}}^{I T} \mathbf{n} \dot{\phi}^{I} \mathrm{~d} I .
\end{aligned}
$$

The displacement in the fluid must match the displacement in the solid, hence

$$
u_{N}=\mathbf{n}^{T} \mathbf{u}^{I}
$$

which means

$$
\begin{aligned}
& \int \frac{1}{\beta} \bar{P}_{0} P_{0} \mathrm{~d} F-\int \frac{\rho_{F}}{\beta} \bar{P}_{0} \dot{\phi} \mathrm{d} F+\int \frac{\rho_{F}}{\beta} \bar{\phi} \dot{P}_{0} \mathrm{~d} F \\
& \quad-\int \frac{\rho_{F}^{2}}{\beta} \bar{\phi} \ddot{\phi} \mathrm{d} F-\int \rho_{F} \nabla \bar{\phi} \cdot \nabla \phi \mathrm{d} F=\int \bar{P}_{0} \mathbf{n}^{T} \mathbf{u}^{I} \mathrm{~d} I \\
& \quad+\int \rho_{F} \bar{\phi}^{I} \mathbf{n}^{T} \dot{\mathbf{u}}^{I} \mathrm{~d} I .
\end{aligned}
$$

Equations (7) and (9) form a complete virtual work statement of the fluid-structure interaction problem.

\subsection{Finite element discretization}

We discretize the virtual work expressions using standard isoparametric interpolation functions[22]. Take

$$
\begin{aligned}
& x=\mathbf{h} \mathbf{x}, \\
& \mathbf{h}-\left[h_{1} h_{2} h_{3} \ldots h_{N}\right] \text {, } \\
& \mathbf{x}^{T}=\left[\begin{array}{lllll}
x_{1} & x_{2} & x_{3} & \ldots & x_{N}
\end{array}\right],
\end{aligned}
$$

where $x=x$ coordinate of a point within an element, $h_{i}=$ interpolation function for node $i, x_{i}=$ $x$ coordinate of node $i$, and $N=$ number of nodes per finite element. Similarly,

$$
\begin{aligned}
y & =\mathbf{h} \mathbf{y}, \\
z & =\mathbf{h ~ z} .
\end{aligned}
$$

For each solid element,

$$
\mathbf{u}=\mathbf{H} \mathbf{U},
$$

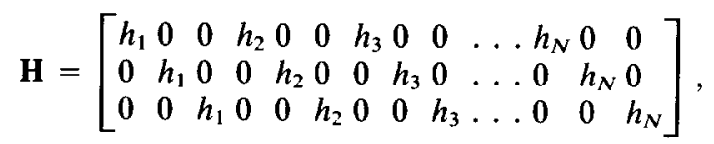

$\mathbf{U}^{T}=\left[\begin{array}{llllllllllll}u_{1} & v_{1} & w_{1} & u_{2} & v_{2} & w_{2} & u_{3} & v_{3} & w_{3} & \ldots & u_{N} v_{N} & w_{N}\end{array}\right]$

where $u_{i}=x$-direction displacement of node $i, v_{i}$ $=y$-direction displacement of node $i$, and $w_{i}=$ $z$-direction displacement of node $i$. For fluid elements,

$$
\begin{gathered}
\phi=\mathbf{h} \boldsymbol{\phi}, \\
\phi^{T}=\left[\begin{array}{lll}
\phi_{1} & \phi_{2} & \phi_{3}
\end{array} \ldots \phi_{N}\right],
\end{gathered}
$$

where $\phi_{i}=$ velocity potential at node $i$.
Now we define matrices for each finite element. The mass and stiffness matrices for the solid $\mathbf{K}_{s s}$ and $\mathbf{M}_{s s}$ take their usual form. The fluid matrices become (as in heat transfer analysis[22])

$$
\begin{aligned}
& \mathbf{M}_{F F}=\int \frac{\rho_{F}^{2}}{\boldsymbol{\beta}} \mathbf{h}^{T} \mathbf{h} \mathrm{d} f, \\
& \mathbf{K}_{F F}=\int \rho_{F} \mathbf{D}^{T} \mathbf{D} \mathrm{d} f,
\end{aligned}
$$

where $\mathrm{d} f$ denotes integration over the fluid element domain (we now use lower case letters to denote element integrations) and where

$$
\mathbf{D}=\left[\begin{array}{l}
\frac{\partial h_{1}}{\partial x} \frac{\partial h_{2}}{\partial x} \frac{\partial h_{3}}{\partial x} \ldots \frac{\partial h_{N}}{\partial x} \\
\frac{\partial h_{1}}{\partial y} \frac{\partial h_{2}}{\partial y} \frac{\partial h_{3}}{\partial y} \ldots \frac{\partial h_{N}}{\partial y} \\
\frac{\partial h_{1}}{\partial z} \frac{\partial h_{2}}{\partial z} \frac{\partial h_{3}}{\partial z} \ldots \frac{\partial h_{N}}{\partial z}
\end{array}\right] .
$$

The fluid-structure coupling matrix for the fluid is

$$
\mathbf{C}_{F S}=-\int \rho_{F} \mathbf{a}^{T} \mathbf{b} \mathbf{d} i,
$$

where $\mathbf{a}=\left[h_{1} h_{2} \ldots h_{M}\right], \mathbf{b}=\left[h_{1} n_{x} h_{1} n_{y} h_{1} n_{z} h_{2} n_{x}\right.$ $\left.h_{2} n_{y} h_{2} n_{z} \ldots h_{M} n_{x} h_{M} n_{y} h_{M} n_{z}\right]$, and $M=$ number of nodes per fluid-structure interface element. The fluid-structure coupling matrix for the solid is $\mathbf{C}_{F S}^{T}$. We also have matrices corresponding to $P_{0}$ (one node per fluid region). They are

$$
\begin{aligned}
& \mathbf{K}_{P P}=-\int \frac{1}{\beta} \mathrm{d} f, \\
& \mathbf{K}_{P S}=\int \mathbf{b} \mathrm{d} i, \\
& \mathbf{C}_{P F}=\int \frac{\rho_{F}}{\beta} \mathbf{h} \mathrm{d} f .
\end{aligned}
$$

We evaluate $\mathbf{K}_{S S}$ and $\mathbf{M}_{S S}$ for solid elements. $\mathbf{M}_{F F}, \mathbf{K}_{F F}, \mathbf{K}_{P P}$, and $\mathbf{C}_{P F}$ are calculated for fluid elements. "Fluid-structure interface elements" enforce the coupling between the fluid and solid regions through the $\mathbf{C}_{F S}$ and $\mathbf{K}_{P S}$ matrices. (If we omit the fluid-structure interface elements, the solid will behave as if there is no fluid and the fluid will act as though the solid is rigid.)

We sum over all element matrices to form global matrices. Changing the sign on the $P_{0}$ equations gives

$$
\begin{gathered}
{\left[\begin{array}{lll}
\mathbf{M}_{\mathrm{SS}} & \mathbf{0} & \mathbf{0} \\
\mathbf{0} & -\mathbf{M}_{F F} & \mathbf{0} \\
\mathbf{0} & \mathbf{0} & \mathbf{0}
\end{array}\right]\left[\begin{array}{l}
\ddot{\mathbf{U}} \\
\ddot{\mathbf{\phi}} \\
\mathbf{P}_{\mathbf{0}}
\end{array}\right]+\left[\begin{array}{lll}
\mathbf{0} & \mathbf{C}_{F S}^{T} & \mathbf{0} \\
\mathbf{C}_{F S} & \mathbf{0} & \mathbf{C}_{P F}^{T} \\
\mathbf{0} & \mathbf{C}_{P F} & \mathbf{0}
\end{array}\right]\left[\begin{array}{l}
\dot{\mathbf{U}} \\
\dot{\boldsymbol{\phi}} \\
\dot{\mathbf{P}}_{\mathbf{0}}
\end{array}\right]} \\
+\left[\begin{array}{lll}
\mathbf{K}_{\mathrm{SS}} & \mathbf{0} & \mathbf{K}_{P S}^{T} \\
\mathbf{0} & -\mathbf{K}_{F F} & \mathbf{0} \\
\mathbf{K}_{\mathrm{PS}} & \mathbf{0} & \mathbf{K}_{P P}
\end{array}\right]\left[\begin{array}{l}
\mathbf{U} \\
\mathbf{\phi} \\
\mathbf{P}_{0}
\end{array}\right]=\left[\begin{array}{l}
\mathbf{R}_{S} \\
\mathbf{0} \\
\mathbf{0}
\end{array}\right]
\end{gathered}
$$

or

$$
\mathbf{M} \ddot{\mathbf{X}}+\mathbf{C} \dot{\mathbf{X}}+\mathbf{K X}=\mathbf{R},
$$


where $\mathbf{R}_{\mathrm{S}}$ represents external loads on the solid computed as usual.

Sevcral features make the implementation of this method relatively direct. Figure 2 shows a schematic mesh discretization. The eight-node solid elements give $\mathbf{K}_{s S}$ and $\mathbf{M}_{s S}$, the eight-node fluid elements give $\mathbf{K}_{F F}, \mathbf{M}_{F F}, \mathbf{K}_{P P}$, and $\mathbf{C}_{P F}$, and finally $\mathbf{K}_{P S}$ and $\mathbf{C}_{F S}$ correspond only to the three-node fluid-structure interface elements. Assigning degrees of freedom for $\phi$ and $U$ in sequence at an interface node produces a tightly banded $\mathbf{C}$ matrix.

We have implemented the formulation given above in ADINA for lumped or consistent mass discretization. In the example solutions considered later we have always used a consistent mass matrix.

\subsection{Transient analysis}

The transient solution of a fluid-structure problem is obtained by numerically integrating eqn (21). We have implemented the fluid-structure analysis capability in ADINA for use with the implicit Newmark or Wilson- $\theta$ time integration schemes.

Our experience is that the time step $\Delta t$ can largely be selected as usual when employing the implicit integration methods[22]. However, a detailed accuracy analysis of various time integration methods applied to eqn (21) would be valuable. This study should also include the effect of the differences in magnitude of the matrix elements in the coefficient matrices as regards round-off errors in the solution of the equations without pivoting. Our experiences are that in practice these errors are usually small but there can be situations when specific care must be taken to number the equations in an appropriate order or an equation solver with a pivoting strategy must be employed.

\subsection{Frequency analysis}

We expect all of the eigenvalues of the fluidstructure system to be real, since physically there is no dissipation in the system. By definition $\dot{\mathbf{U}}=$ $\boldsymbol{\nabla} \phi$, so we assume that $\mathbf{U}=\check{\mathbf{U}} \sin (\omega t)$ and $\boldsymbol{\phi}=$ $\tilde{\phi} \cos (\omega t)$. Since pressure is $P_{0}-\rho_{F} \dot{\phi}$ we take $\mathbf{P}_{0}$ $=\overline{\mathbf{P}} \sin (\omega t)$ to give

$$
\begin{array}{r}
\mathbf{K}_{S S} \tilde{\mathbf{U}} \sin (\omega t)+\mathbf{K}_{P S}^{T} \tilde{\mathbf{P}} \sin (\omega t)-\omega \mathbf{C}_{F S}^{T} \tilde{\boldsymbol{\phi}} \sin (\omega t) \\
-\omega^{2} \mathbf{M}_{S S} \tilde{\mathbf{U}} \sin (\omega t)=\mathbf{0} \\
-\mathbf{K}_{F F} \tilde{\boldsymbol{\phi}} \cos (\omega t)+{ }^{-} \mathbf{C}_{F S} \tilde{\mathbf{U}} \cos (\omega t)+\omega \mathbf{C}_{P F}^{T} \tilde{\mathbf{P}} \\
\times \cos (\omega t)+\omega^{2} \mathbf{M}_{F F} \tilde{\boldsymbol{\phi}} \cos (\omega t)=\mathbf{0} \\
\mathbf{K}_{P S} \tilde{\mathbf{U}} \sin (\omega t)+\mathbf{K}_{P P} \overline{\mathbf{P}} \sin (\omega t) \\
-\omega \mathbf{C}_{P F} \overline{\boldsymbol{\phi}} \sin \omega t=\mathbf{0}
\end{array}
$$

Changing the sign on the fluid equation (24) gives

$$
\begin{array}{r}
{\left[\begin{array}{lll}
\mathbf{K}_{S S} \mathbf{0} & \mathbf{K}_{P S}^{T} \\
\mathbf{0} & \mathbf{K}_{F F} & \mathbf{0} \\
\mathbf{K}_{P S} \mathbf{0} & \mathbf{K}_{P r}
\end{array}\right]\left[\begin{array}{l}
\tilde{\mathbf{U}} \\
\tilde{\mathbf{\phi}} \\
\tilde{\mathbf{P}}
\end{array}\right]-\omega\left[\begin{array}{lll}
\mathbf{0} & \mathbf{C}_{F S}^{T} & \mathbf{0} \\
\mathbf{C}_{F S} & \mathbf{0} & \mathbf{C}_{P F}^{T} \\
\mathbf{0} & \mathbf{C}_{P F} & \mathbf{0}
\end{array}\right]\left[\begin{array}{l}
\mathbf{U} \\
\tilde{\mathbf{\phi}} \\
\tilde{\mathbf{P}}
\end{array}\right]} \\
-\omega^{2}\left[\begin{array}{lll}
\mathbf{M}_{S S} \mathbf{0} & \mathbf{0} \\
\mathbf{0} & \mathbf{M}_{F F} \mathbf{0} \\
\mathbf{0} & \mathbf{0} & \mathbf{0}
\end{array}\right]\left[\begin{array}{l}
\tilde{\mathbf{U}} \\
\tilde{\mathbf{\phi}} \\
\tilde{\mathbf{P}}
\end{array}\right]=\mathbf{0}
\end{array}
$$

We now have a real symmetric eigenproblem of the form

$$
\mathbf{K}^{\prime} \tilde{\mathbf{X}}-\omega \mathbf{C}^{\prime} \tilde{\mathbf{X}}-\omega^{2} \mathbf{M}^{\prime} \tilde{\mathbf{X}}=\mathbf{0}
$$

To solve this eigenproblem, we could have researched a modification of the subspace iteration method or the determinant search method[22].

We established three properties (see Appendix A) for this eigenproblem which enabled us to modify the standard determinant search method in ADINA[22]. First, all of the eigenvalues $\omega^{2}$ are real and greater than or equal to zero. Second, if we factorize

$$
\mathbf{K}^{*}=\mathbf{L} \mathbf{D} \mathbf{L}^{\prime}=\mathbf{K}^{\prime} \quad \mu \mathbf{C}^{\prime}-\mu^{2} \mathbf{M}^{\prime}
$$

where $\mathbf{L}$ is a lower triangular matrix with ones on its diagonal and $\mathbf{D}$ is a diagonal matrix, then the number of negative elements in $\mathbf{D}$ is equal to the number of eigenvalues below $\mu^{2}$ plus the number of variables in $\tilde{\mathbf{P}}$. (Note that the number of $\tilde{\mathbf{P}}$ variables is equal to the number of negative diagonal elements in $\mathbf{K}^{\prime}$.) Third, the eigenvectors $\tilde{\mathbf{X}}_{t}$ satisfy the orthogonality relation

$$
\mathbf{0}=\tilde{\mathbf{X}}_{i}^{l} \mathbf{C}^{\prime} \overline{\mathbf{X}}_{j}+\left(\omega_{i}+\omega_{j}\right) \overline{\mathbf{X}}_{i}^{l} \mathbf{M}^{\prime} \ddot{\mathbf{X}}_{j} ; \quad i \neq j
$$

Hence, the procedure for the determinant search solution contains the following steps:

(1) Perform secant iteration on $p(\lambda)=\operatorname{det}\left[\mathbf{K}^{\prime}-\right.$ $\left.\sqrt{\lambda} \mathbf{C}^{\prime}-\lambda \mathbf{M}^{\prime}\right]$ until we are near a root $\lambda_{i}(=$ $\left.\omega_{i}^{2}\right)$. (i) Estimate $\lambda_{i}$ using the secant formula. (ii) Form $\mathbf{K}^{*}=\mathbf{L} \mathbf{D} \mathbf{L}^{T}$ at that estimate. (iii) Count the negative elements in $\mathbf{D}$ to find the number of eigenvalues below the estimate for $\lambda_{i}$.

(2) Perform an inverse iteration on $\mathbf{K} * \dot{\mathbf{X}}=1 \omega^{2}$ $-\lambda)\left(f, \mathbf{C}^{\prime} \tilde{\mathbf{X}}+\mathbf{M}^{\prime} \tilde{\mathbf{X}}\right)$, where $\lambda$ is the estimate for $\lambda_{i}$ and $f_{c}=(\omega-\sqrt{\lambda}) /\left(\omega^{2}-\lambda\right)$, to simultaneously identify a solution for $\omega$ and $\bar{X}$. (i) Update $f$, in each iteration.

(3) Orthogonalize (approximately) the current eigenvector guess to previously calculated eigenvectors. (i) Use the orthogonality relation (29), with the current guess for $\omega_{i}$, with one eigenvector at a time. (ii) Orthogonalize to (up to) last seven eigenvectors. (iii) Operate on most recently found eigenvector last.

This iteration procedure is not substantially different from the determinant search procedure without a $\mathbf{C}$ matrix[22]. All operations are in real arithmetic.

\section{OLD FXAMPI,F PROBI,FMS}

In our previous paper[1] we defined four categories of fluid-structure interaction problems (review Fig. 1). As mentioned previously, we were able to solve problems from categories 1,2 , and 3 . but we were unable to solve category 4 problems (solids vibrating in incompressible fluids). In this 


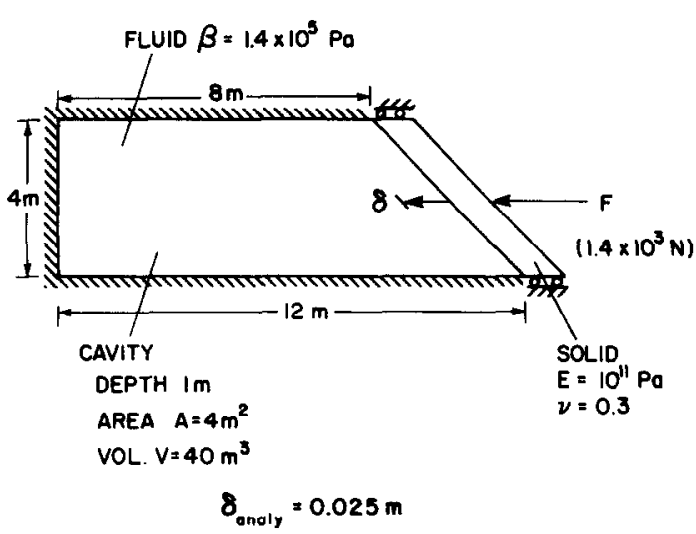

Fig. 3. Tilted piston-container test case.

section we attempt to solve problems from each category with the $\phi-U-P_{0}$ formulation.

Figure 3 shows the tilted piston-container test case from category 1 (static analyses). Figure 4 shows the mesh and results. The $\phi-U-P_{0}$ formulation solution is as accurate as the displacementbased formulation without the rotation penalty. Displacement-based elements including the rotation penalty require many more elements for an accurate solution. Since the formulation is derived from a velocity potential, the $\phi-U-P_{0}$ solution is inherently irrotational.

Figures 5 and 6 show a test case from category 2 , the tall water column problem. Since the walls are rigid and the pressure at the free surface is zero, the $\phi-U-P_{0}$ formulation reduces to an ordinary acoustic fluid formulation ( $\phi$ only). Table 1 shows the excellent results obtained. (We used this mesh in our earlier paper[1]. We could have chosen a coarser mesh.)

Category 3 involved fluids in flexible boundaries, and Fig. 7 shows the piston-container test problem. Figure 8 shows the finite element model.

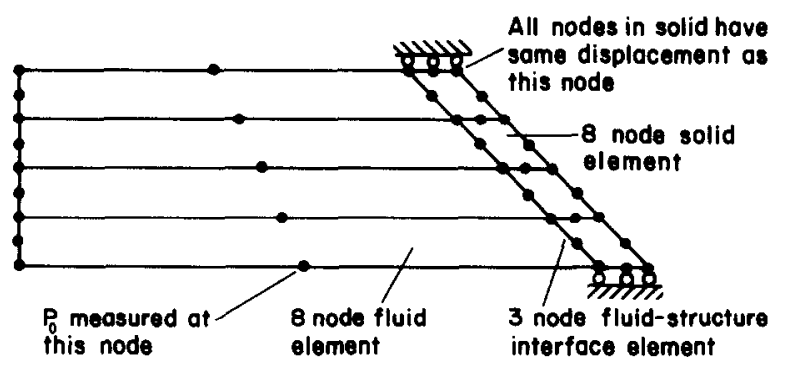

$\begin{array}{lc}\begin{array}{lc}\text { Method } \\ \begin{array}{l}\phi-U-P_{0} \\ \text { (shown obove) }\end{array}\end{array} & \frac{8}{0.025} \\ \begin{array}{l}\text { Displacements without rotation } \\ \text { Denalty }\end{array} & 0.025 \\ \begin{array}{l}\text { Displacements with rotation } \\ \text { penalty }\end{array} & 0.016\end{array}$

Fig. 4. Tilted piston-container mesh and results.

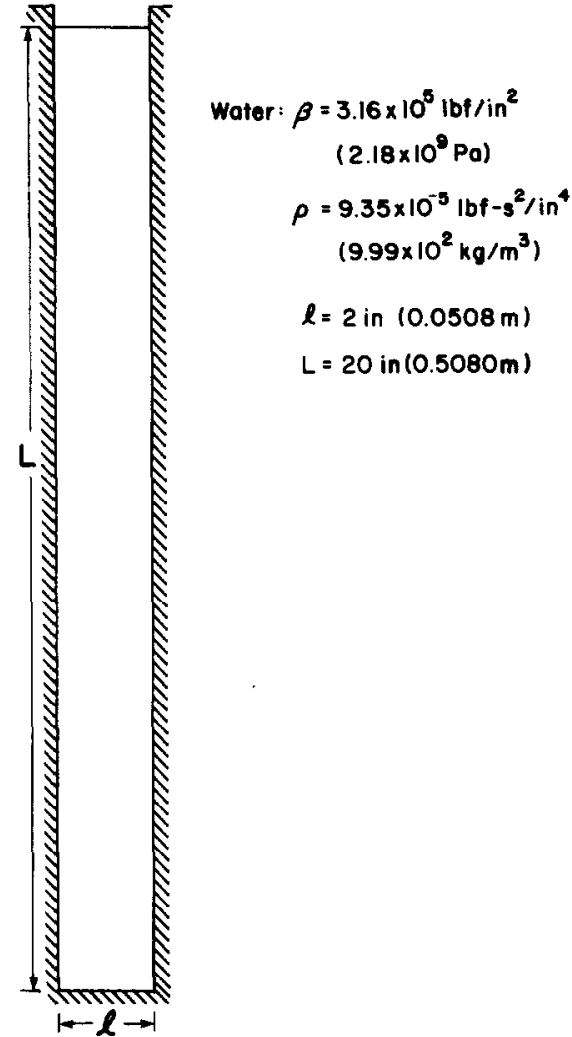

Fig. 5. Tall water column problem.

Table 2 summarizes the results, which are again excellent.

The category 4 problem is the most significant, since we could not solve it adequately using displacement-based elements. Figures 9 and 10 show the physical problem (the ellipse-on-spring problem) and the finite element discretization. The analytical first frequency of the system is $28.2 \mathrm{rad} / \mathrm{sec}$. Using 60 displacement-based fluid elements with full integration, we found a predicted frequency of over $3000 \mathrm{rad} / \mathrm{sec}$. With the $\phi-U-P_{0}$ formulation and full integration, we calculated a frequency of $27.8 \mathrm{rad} / \mathrm{sec}$. We suspect that the finite element frequency is lower than the analytical value because the ellipse shape is not modeled exactly. Figure 11 shows calculated frequencies for several small adjustments to the ellipse shape, which seem to indicate that the ellipse shape influences the predicted frequency enough to explain the $2 \%$ underestimate.

Table 1. Analytical and finite element solutions to natural frequencies of tall water column

\begin{tabular}{ccc}
$\begin{array}{c}\text { Mode } \\
\text { number }\end{array}$ & $\begin{array}{c}\text { Analytical solution } \\
(\mathrm{rad} / \mathrm{sec})\end{array}$ & $\begin{array}{c}\text { Finite element } \\
\text { solution } \\
(\mathrm{rad} / \mathrm{sec})\end{array}$ \\
\hline 1 & 4566 & 4567 \\
2 & 13698 & 13730 \\
3 & 22830 & 22980 \\
\hline
\end{tabular}




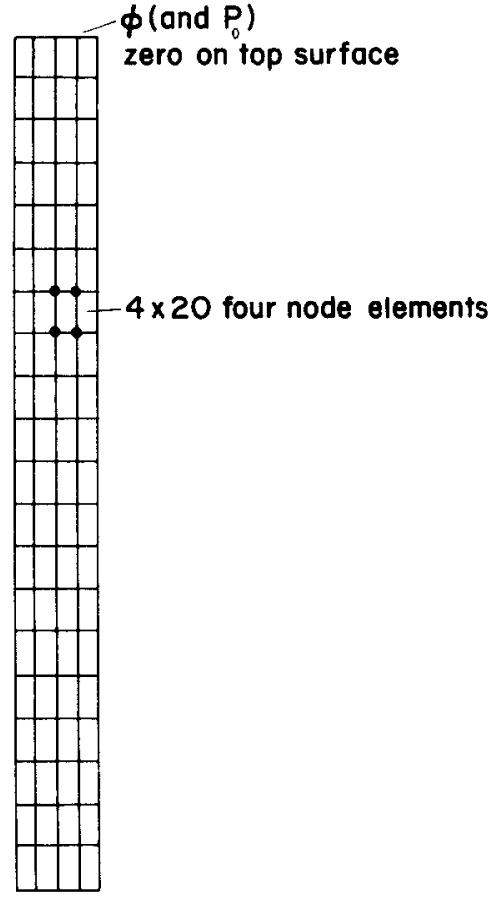

Fig. 6. Finite element mesh used for tall water column.

\section{NEW EXAMPLE PROBLEMS}

\subsection{Transient analysis}

First we solved a simple one-dimensional problem using the displacement formulation and the $\phi-$ $U-P_{0}$ formulation. Figure 12 shows the test case, in which a piston slowly compresses a column of fluid, and also shows the 10-element models. As shown in Fig. 13, the results compare favorably. (All results were obtained using Newmark's method with $\delta=0.5$ and $\alpha=0.25$, the trapezoidal rule[22].)

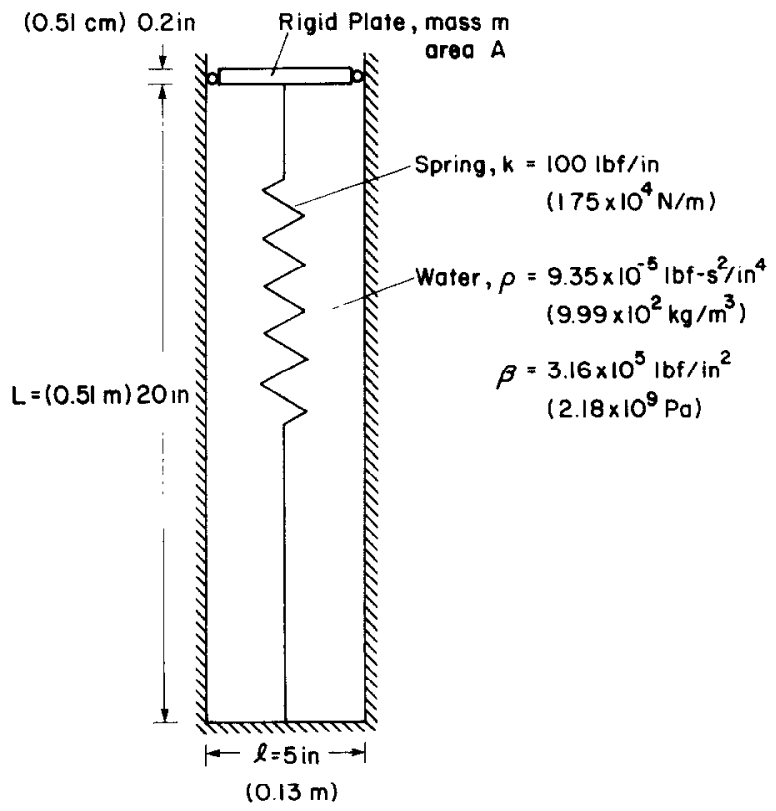

Fig. 7. Piston-container problem.

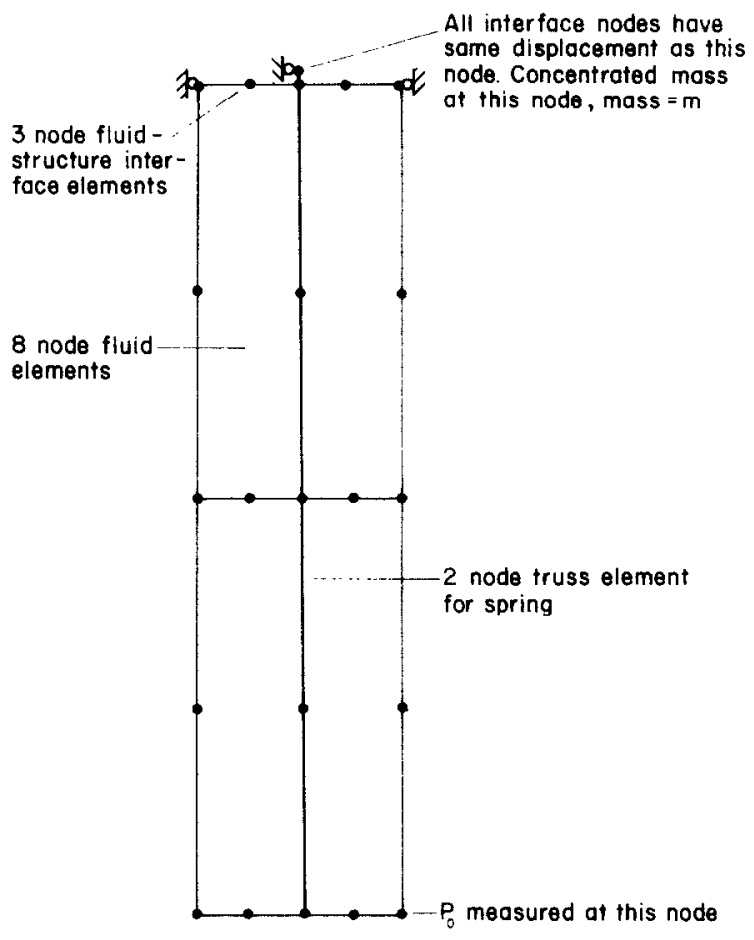

Fig. 8. Finite element model of piston-container problem.

The plate-on-spring in box (Fig. 14) is similar to the piston-container problem solved previously. To start the analysis. we impose a uniform initial displacement on the solid and a corresponding pressure change in $P_{0}$. These initial conditions cause

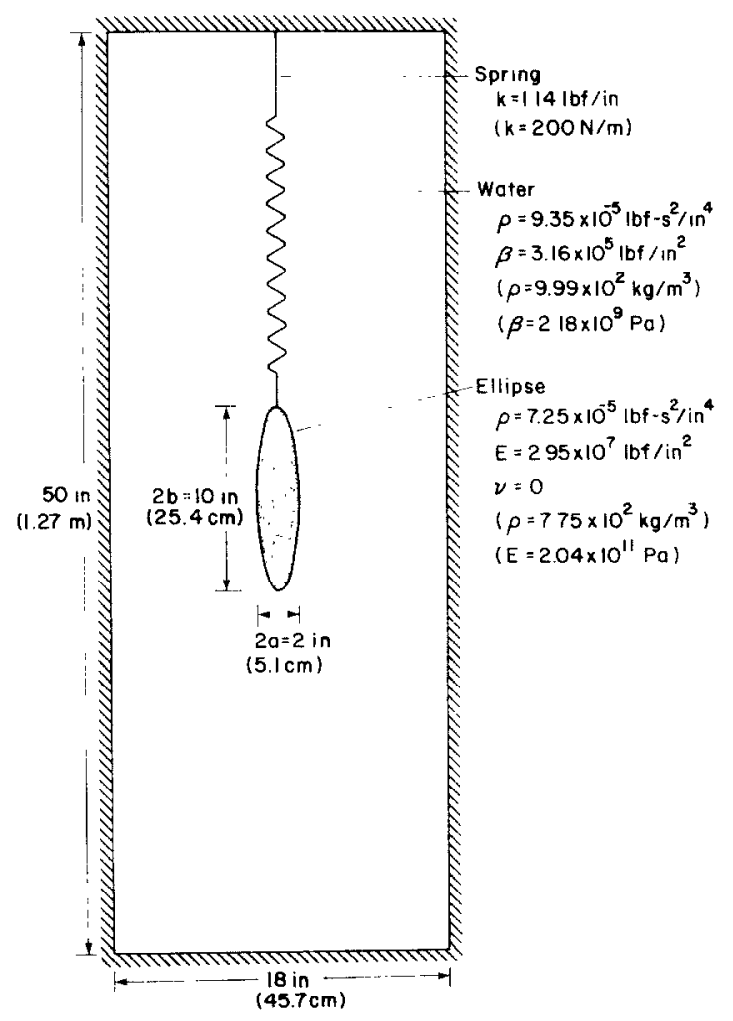

Fig. 9. Ellipse-on-spring problem. 
Table 2. Analytical and finite element solutions to piston-container problem

\begin{tabular}{lccc}
\hline $\begin{array}{c}\text { Piston } \\
\text { mass }\end{array}$ & $\begin{array}{c}\text { Frequency in } \\
\text { vacuum } \\
(\mathrm{rad} / \mathrm{sec})\end{array}$ & $\begin{array}{c}\text { Analytical frequency in } \\
\text { water } \\
(\mathrm{rad} / \mathrm{sec})\end{array}$ & $\begin{array}{c}\text { Finite element } \\
\text { frequency in } \\
\text { water } \\
(\mathrm{rad} / \mathrm{sec})\end{array}$ \\
\hline 1.0 & 10 & 281 & 281 \\
0.1 & 31.6 & 876 & 876 \\
0.01 & 100 & 2441 & 2441 \\
0.001 & 316 & 4130 & 4131 \\
\hline
\end{tabular}

the system to vibrate at a frequency near the fundamental frequency of the system. Figure 14 shows the system and finite element model, while Fig. 15 shows the finite element solution.

In the circle-on-spring in box problem (Fig. 16) the fluid behaves nearly incompressibly. If we impose an initial displacement on the circle, the system will vibrate at approximately its fundamental natural frequency. We could not adequately solve this type of transient problem using displacementbased fluid finite elements. Figure 17 shows the transient solution for this problem using the $\phi_{-} U_{-}$ $P_{0}$ formulation.

\subsection{Frequency analysis}

4.2.1 Convergence. Our finite element frequency results for the $\phi-U-P_{0}$ formulation generally converge from above. Table 3 shows results for various meshes for the plate-on-spring in box problem. Table 4 shows results for the circle-on-spring

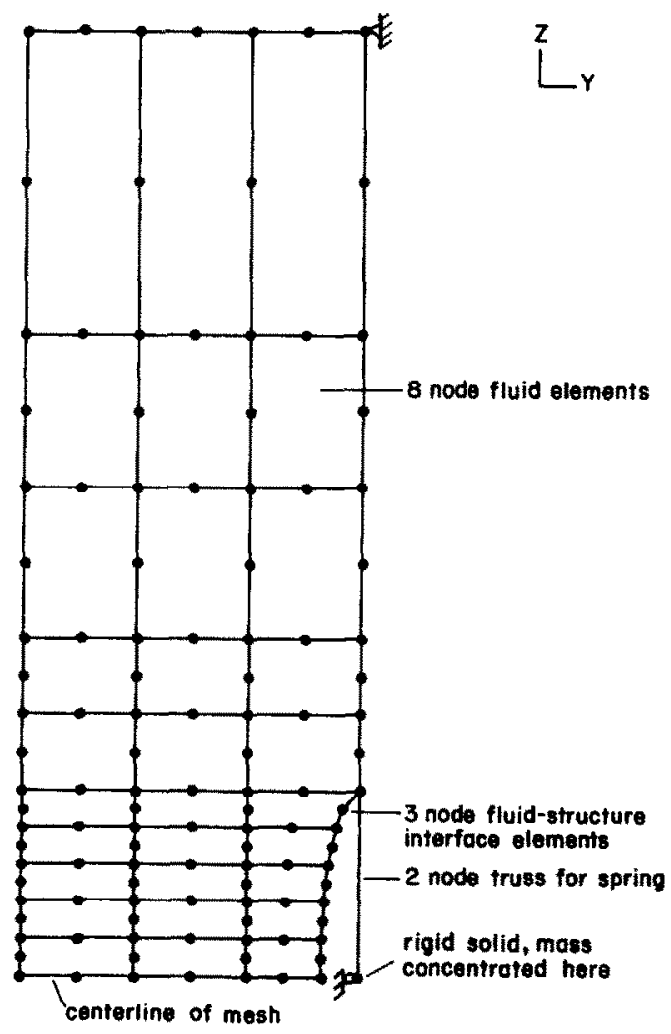

Fig. 10. Mesh used to model ellipse-on-spring. in box problem. In each case convergence is quite rapid.

4.2.2 Plate in water. Sundqvist[24] modeled a 3D plate in water using displacement-based fluid finite elements, and compared finite element frequencies with experimental data. Figure 18 shows the physical problem. Table 5 gives experimental results for natural frequencies of the plate in air and water (from Sundqvist).

Following Sundqvist, we modeled one quarter of the physical domain with three-dimensional finite elements. We used 20-node continuum elements in both the fluid and the solid. Full integration was used throughout. Figures 19 and 20 show the plate in vacuum and plate in water meshes.

For the plate in vacuum, we calculated the frequencies shown in Table 6. All of the finite element

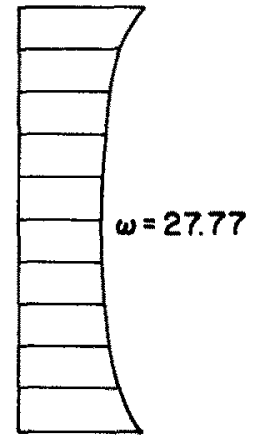

Original Ellipse Mesh (elements near ellipse shown)

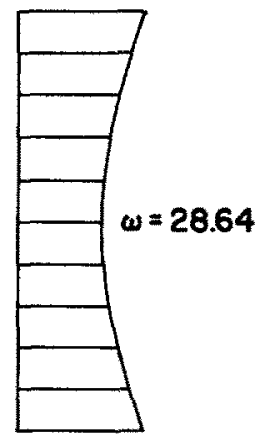

Alternate Mesh 2

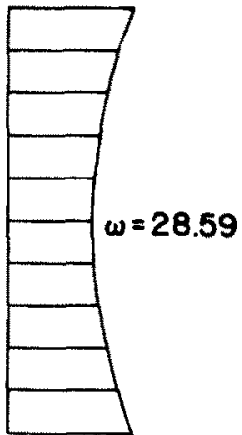

Alternate Mesh I

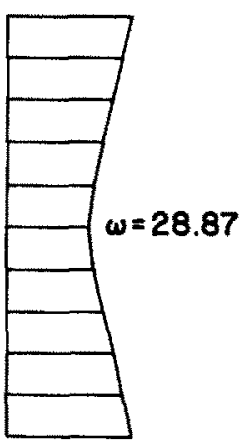

Alternate Mesh 3
Fig. 11. Finite element frequencies for various ellipse-like boundaries (analytical frequency 28.2). 

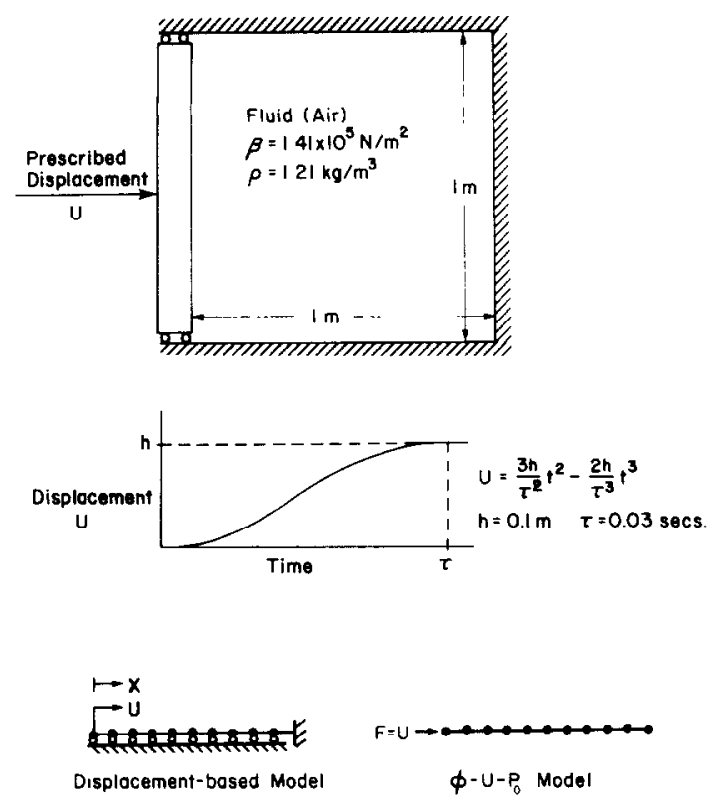

Fig. 12. Transient column compression test case.

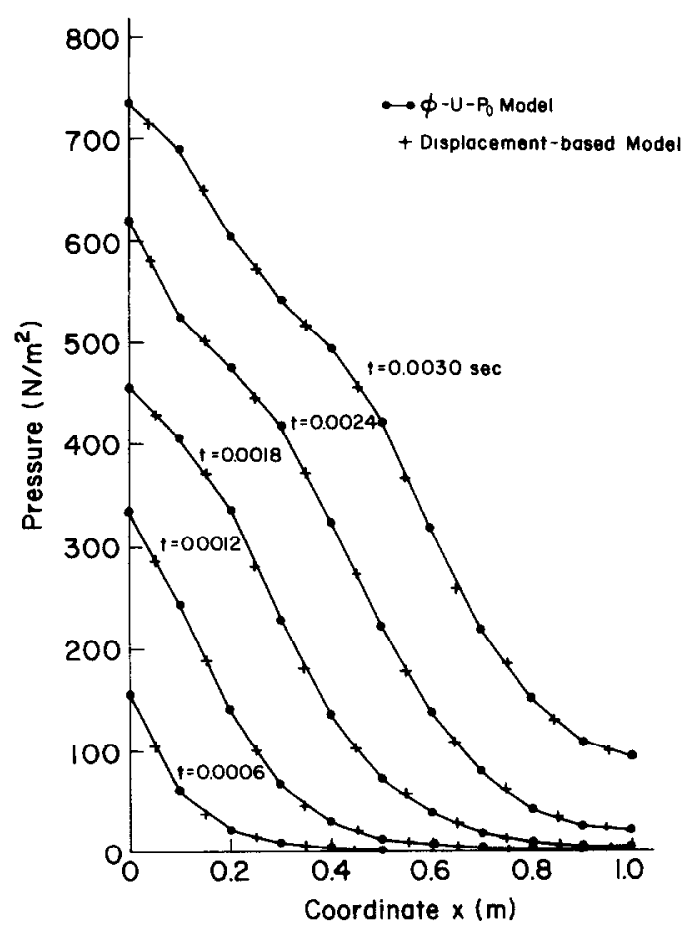

Fig. 13. Results for transient column compression test case.

Table 3. Finite element frequencies for plate-on-spring in box with various numbers of fluid elements

$\left(\omega_{\text {analytical }}=211.3 \mathrm{rad} / \mathrm{sec}\right)$

\begin{tabular}{cc}
\hline \multicolumn{2}{c}{$\left(\omega_{\text {analytical }}=211.3 \mathrm{rad} / \mathrm{sec}\right)$} \\
Fumber of fluid elements & Frequency $(\mathrm{rad} / \mathrm{sec})$ \\
\hline 1 & 211.8 \\
2 & 211.3 \\
3 & 211.3 \\
4 & 211.3
\end{tabular}

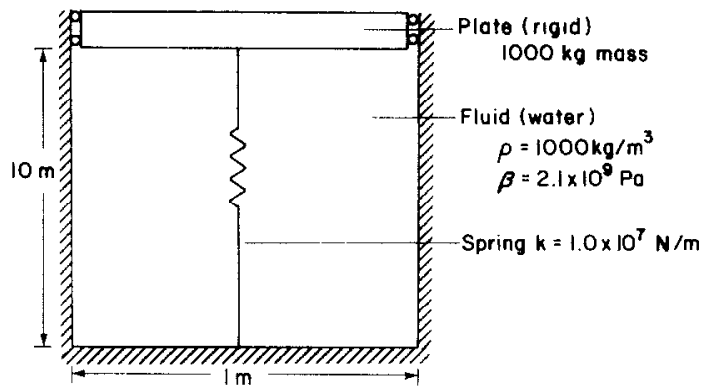

System

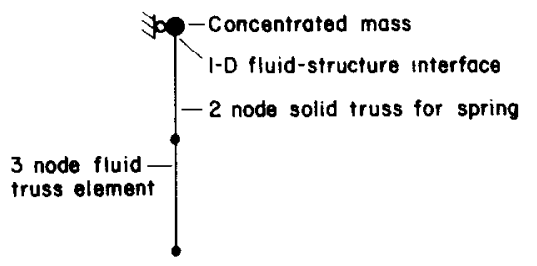

Finite Element Model

Fig. 14. System and finite element model for plate-onspring in box.

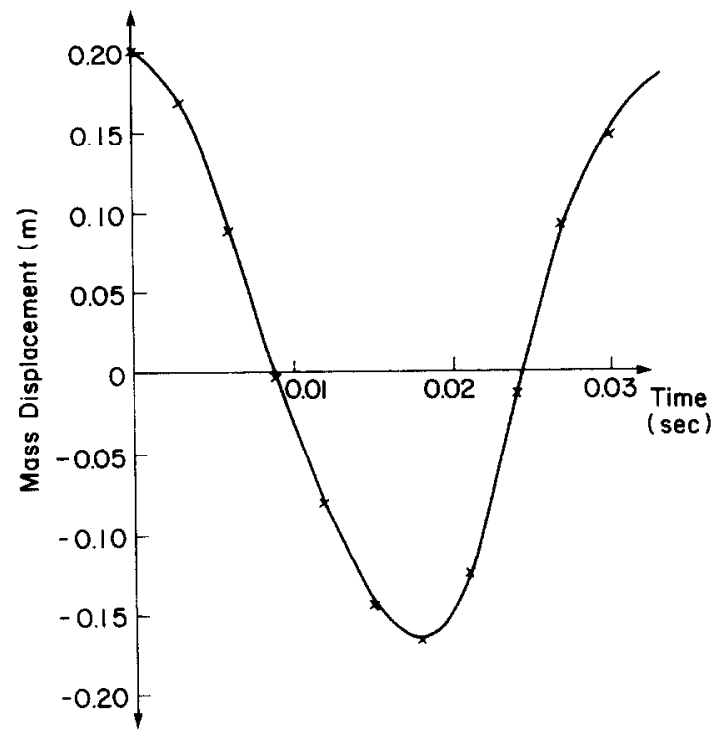

Fig. 15. Transient response of finite element model for plate-on-spring in box (analytical period $0.030 \mathrm{sec}$ ).

Table 4. Finite element frequencies for circle-on-spring in box with various numbers of fluid elements

\begin{tabular}{cc}
\hline \multicolumn{2}{c}{$\left(\omega_{\text {analytical }}=4.44 \mathrm{rad} / \mathrm{sec}\right)$} \\
Number of fluid elements & Frequency $(\mathrm{rad} / \mathrm{sec})$ \\
\hline 8 & 4.89 \\
32 & 4.57 \\
128 & 4.45 \\
\hline
\end{tabular}


Analysis of fluid-structure interactions. A direct symmetric coupled formulation

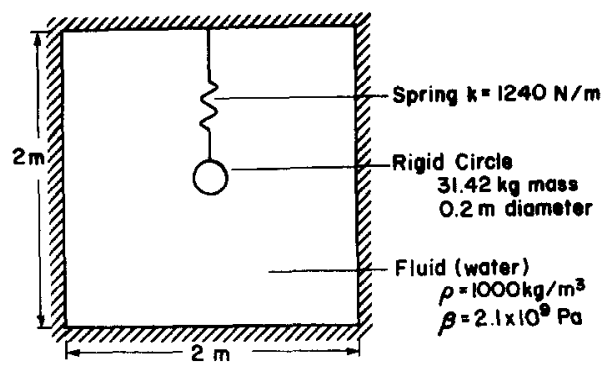

System

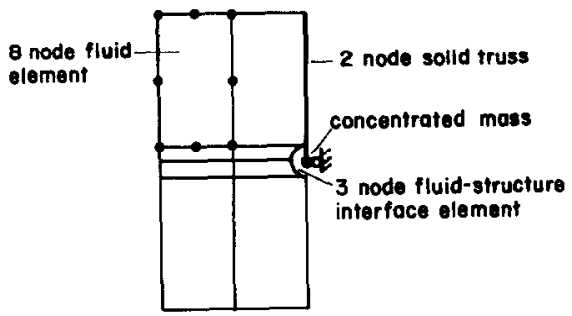

Finite Element Model

Fig. 16. System and finite element model for circle-onspring in box.

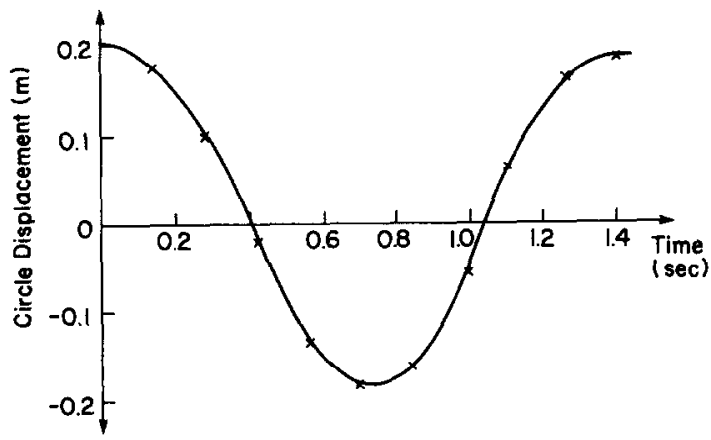

Fig. 17. Transient response of finite element model for circle-on-spring in box (analytical period $1.42 \mathrm{sec}$ ).

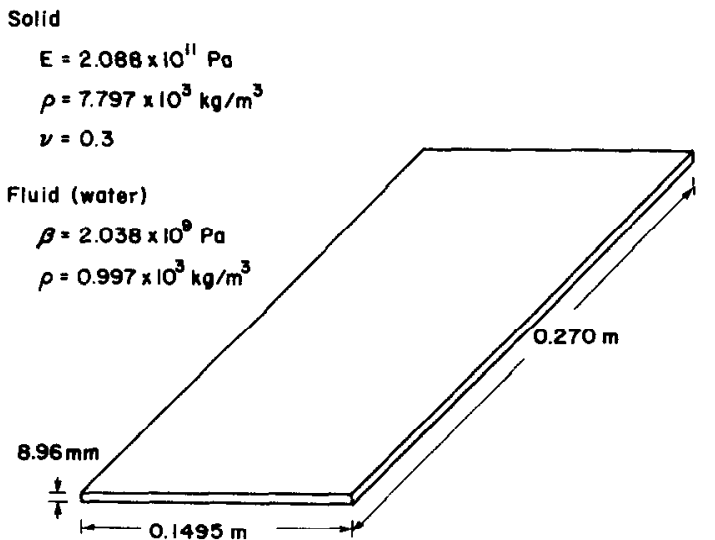

Fig. 18. Sundqvist's plate.

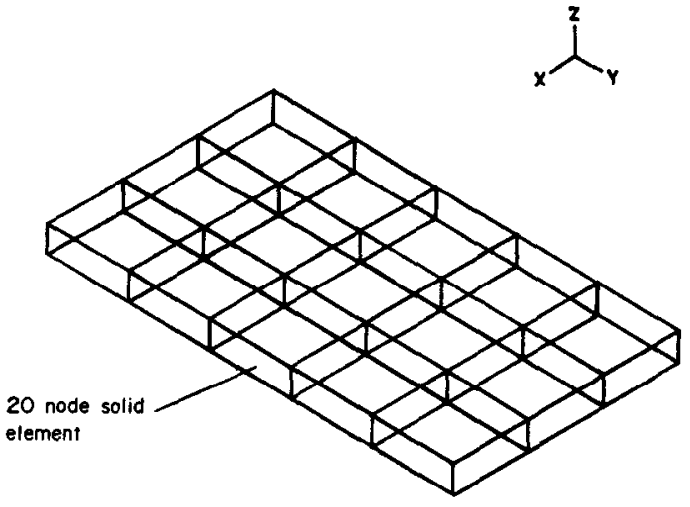

Fig. 19. Finite element mesh for 3D plate in vacuum.

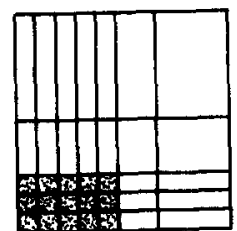

20 node solid element

20 node fluid element

(a)
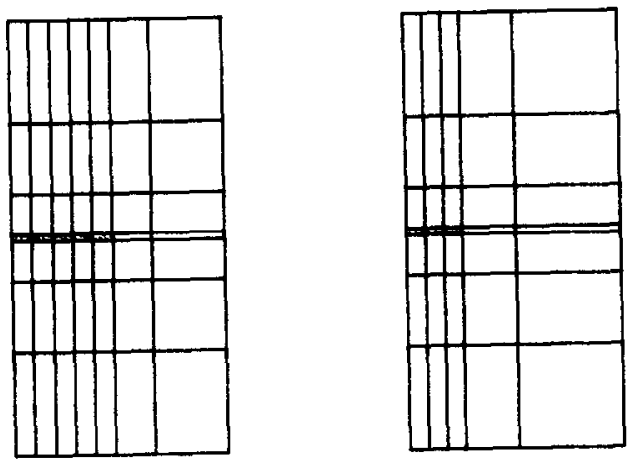

(c)

Fig. 20. Finite element mesh for 3D plate in water. (a) Top view; (b) front view; (c) side view.

results are between $2.1 \%$ and $2.8 \%$ higher than the experimental results.

Ideally, the box around the fluid should be two or three characteristic lengths away from the solid, while at the same time the lowest natural frequency of the fluid alone in the box should be two or three times the important fluid-structure frequencies. We

Table 5. Experimental data for frequencies of free plate in air and water [Ref. 24]

\begin{tabular}{cc}
\hline $\begin{array}{c}\text { Frequencies in air } \\
(\mathrm{Hz})\end{array}$ & $\begin{array}{c}\text { Frequencies in water } \\
(\mathrm{Hz})\end{array}$ \\
\hline 641 & 497 \\
712 & 575 \\
1577 & 1293 \\
1766 & 1408 \\
2139 & 1758 \\
\hline
\end{tabular}


Table 6. Finite element and experimental data for plate in vacuum

\begin{tabular}{ccc}
\hline $\begin{array}{c}\text { Finite element } \\
\text { frequency } \\
(\mathrm{Hz})\end{array}$ & $\begin{array}{c}\text { Experimental } \\
\text { frequency } \\
(\mathrm{Hz})\end{array}$ & $\begin{array}{c}\text { Difference } \\
\text { (percent) }\end{array}$ \\
\hline 656 & 641 & 2.3 \\
727 & 712 & 2.1 \\
1618 & 1577 & 2.6 \\
1815 & 1766 & 2.8 \\
2192 & 2139 & 2.5 \\
\hline
\end{tabular}

cannot meet both these criteria simultaneously for this problem. Instead we chose a $0.570 \times 0.570 \times$ 0.570 meter box which is one characteristic length away from the solid and has a lowest fluid frequency of $1255 \mathrm{~Hz}$. We expect to find purely fluid frequencies along with the fluid-structure interaction frequencies.

Using symmetric-symmetric boundary conditions we found the finite element frequencies listed in Fig. 21. Figure 21 also shows the first mode shape of the submerged plate. The two plate frequencies are $3.9 \%$ and $4.9 \%$ high showing good agreement with the experimental data. The first symmetricsymmetric fluid frequency lies within $1 \%$ of the analytical value for the fluid with no plate.

Some important differences exist between our model and Sundqvist's model. We used continuum elements to model the plate, while Sundqvist used nine-node plate/shell elements. The $\phi-U-P_{0}$ model contains no unphysical solutions, whereas Sundqvist encountered hundreds of zero modes and some additional spurious modes in the range of in-

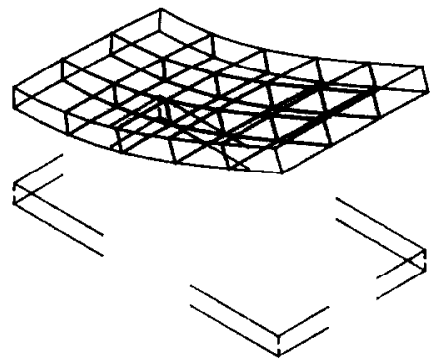

MODE SHAPE CORRESPONDING TO $516.3 \mathrm{HZ}$

\section{SYMMETRIC-SYMMETRIC PLATE}

\section{IN WATER}

$\begin{array}{ccc}\text { F.E. FREO. (Hz) } & \text { EXP. FREO. (Hz) } & \text { DIFF. (X) } \\ 0 & \text { (plate Flold body mode) } & \\ 516.3 & 497 & 3.0 \\ 1243 & \text { fluid mode, } 1255 & -1.0 \\ 1844 & 1758 & 4.9\end{array}$

Fig. 21. Finite element results for 3D plate in water.
Table 7. Comparison of data for $\phi-U-P_{0}$ model and Sundqvist displacement-based model

\begin{tabular}{lrr}
\hline & Sundqvist & \multicolumn{1}{c}{,$U-P_{0}$} \\
\hline Number of equations & 3947 & 1759 \\
Number of matrix & 1034494 & 366270 \\
$\quad$ elements & 2660 & 1759 \\
Maximum half-bandwidth & 263 & 209 \\
Mean half-bandwidth & & \\
\hline
\end{tabular}

terest. Also, Sundqvist had to very judiciously select sliding interfaces in the fluid, while we could model the system directly using our $\phi-U_{-} P_{0}$ formulation.

Table 7 compares the number of degrees of freedom. bandwidth, etc. in the $\phi-U-P_{0}$ formulation model with the displacement-based model of Sundqvist. (Since Sundqvist used eight-node elements in the fluid, his bandwidth is comparable to ours even though he used more than twice as many equations.) Clearly we can obtain good results with much less computational effort using the $\phi-U-P_{4}$ formulation.

\section{CONCLUDING REMARKS}

The $\phi-U-P_{0}$ formulation provides an excellent alternative to displacement-based fluid finite elements. The approach is easy to implement in displacement-based finite element programs, and requires fewer degrees of freedom in the fluid. Static cases give good results with coarse meshes. Transient analyses involve relatively simple extensions of known techniques. Frequency solutions require more modifications, but using the real symmetric properties of the formulation (and the limited fluid degrees of freedom) reduces computing costs considerably.

Acknowledgments-We are grateful for the financial support of ADINA Engineering AB, Sweden, and of the Forschungszentrum GKSS. West Germany, through Mr. R. Dietrich, for this work. We also thank International Business Machines Corporation for providing financial support for Lorraine Olson.

\section{REFERENCES}

1. L. G. Olson and K. J. Bathe, A study of displacementbased fluid finite elements for calculating frequencies of fluid and fluid-structure systems. Nucl. Engng Des. 76, 137-151 (1983).

2. G. C. Everstine, A symmetric potential formulation for fluid-structure interaction. Letter to the Editor. $J$. Sound Vib. 79, 157-160 (1981).

3. G. C. Everstein, M. S. Marcus and A. J. Quezon, Finite element analysis of fluid-filled elastic piping systems. Eleventh NASTRANUser's Colloquium, May 1983, pp. 141-160.

4. H. C. Neilson, G. C. Everstine and Y. F. Wang, Transient response of a submerged fluid-coupled doublewalled shell structure to a pressure pulse. J. Acoust. Soc. Am. 70, 1776-1782 (1981).

5. G. C. Everstine. Structural-acoustic finite element analysis, with application to scattering. Proceedings 
of the 6th Invitational Symposium on the Unification of Finite Elements, Finite Differences, and Calculus of Variations, University of Connecticut, May 1982 , pp. 101-122.

6. O. C. Zienkiewicz and R. E. Newton, Coupled vibrations of a structure submerged in a compressible fluid. Symposium on Finite Element Techniques, Stuttgart, 1969.

7. W. J. T. Daniel, Performance of reduction methods for fluid-structure and acoustic eigenvalue problems. Int. J. Numer. Meth. Engng 15, 1585-1594 (1980).

8. W. J. T. Daniel, Modal methods in finite element fluidstructure eigenvalue problems. Int. J. Numer. Meth. Engng 15, 1161-1175 (1980).

9. M. Petyt and S. P. Lim, Finite element analysis of the noise inside a mechanically excited cylinder. Int. $J$. Numer. Meth. Engng 13, 109-122 (1978).

10. W. C. Müller, Simplified analysis of linear fluid-structure interaction. Int. J. Numer. Meth. Engng 17, 113121 (1981).

11. A. Craggs, The transient response of a coupled plateacoustic system using plate and acoustic finite elements. J. Sound Vib. 15, 509-528 (1971).

12. T. B. Belytschko and J. M. Kennedy, A fluid-structure finite element method for the analysis of reactor safety problems. Nucl. Engng Des. 38, 71-81 (1976).

13. T. B. Belytschko and J. M. Kennedy, Computer models for subassembly simulation. Nucl. Engng Des. 49, 17-38 (1978).

14. D. P. Flanagan and T. B. Belytschko, A uniform strain hexahedron and quadrilateral with orthogonal hourglass control. Int. J. Numer. Meth. Engng 17, 679706 (1981).

15. A. K. Chopra, E. L. Wilson and I. Farhoomand, Earthquake analysis of reservoir-dam systems. Proceedings of the 4th World Conference on Earthquake Engineering, Santiago, Chile, 1969, pp. 1-10.

16. E. L. Wilson and M. Khalvati, Finite elements for the dynamic analysis of fluid-solid systems. Int. J. Numer. Meth. Engng 19, 1657-1668 (1983).

17. K. J. Bathe and W. Hahn, On transient analysis of fluid-structure systems. Comput. Struct. 10, 383-391 (1979).

18. M. A. Hamdi, Y. Ousset and G. Verchery, A displacement method for the analysis of vibrations of coupled fluid-structure systems. Int. J. Numer. Meth. Engng 13, 139-150 (1978).

19. R. Ohayon and R. Valid, True symmetric formulations of free vibrations of fluid-structure interaction. Applications and extension. International Conference on Numerical Methods for Coupled Problems, University of Swansea (U.K.), 1981.

20. O. C. Zienkiewicz and P. Bettess, Fluid-structure dynamic interaction and wave forces. An introduction to numerical treatment. Int. J. Numer. Meth. Engng 13, 1-16 (1978).

21. T. B. Belytschko, Fluid-structure interaction. Comput. Struct. 12, 459-469 (1980).

22. K. J. Bathe, Finite Element Procedures in Engineering Analysis. Prentice-Hall, Englewood Cliffs, N.J. (1982).

23. H. Lamb, Hydrodynamics, 6th Edn. Dover Publications, New York (1945).

24. J. Sundqvist, An application of ADINA to the solution of fluid-structure interaction problems. Comput. Struct. 17, 793-807 (1983).

\section{APPENDIX A}

Three properties of our particular eigenproblem were summarized in Sec. 2.4 of this paper. Here we discuss each property in more detail.

A.1. The eigenvalues are real and greater than or equal to zero
Suppose we have a solution $\overline{\mathbf{X}}$ to the eigenproblem

$$
\mathbf{K}^{\prime} \overline{\mathbf{X}}-\omega \mathbf{C}^{\prime} \overline{\mathbf{X}}-\omega^{2} \mathbf{M}^{\prime} \tilde{\mathbf{X}}=\mathbf{0}
$$

then it must be true that

$$
\tilde{\mathbf{X}}^{T} \mathbf{K}^{\prime} \overline{\mathbf{X}}-\omega \tilde{\mathbf{X}}^{T} \mathbf{C}^{\prime} \overline{\mathbf{X}}-\omega^{2} \overline{\mathbf{X}}^{T} \mathbf{M}^{\prime} \overline{\mathbf{X}}=0 .
$$

Solving for $\omega$ gives

$$
\omega=\frac{b \pm \sqrt{b^{2}+4 a c}}{-2 c}
$$

where

$$
\begin{aligned}
a & =\tilde{\mathbf{X}}^{T} \mathbf{K}^{\prime} \dot{\mathbf{X}}, \\
b & =\tilde{\mathbf{X}}^{T} \mathbf{C}^{\prime} \overline{\mathbf{X}}, \\
c & =\tilde{\mathbf{X}}^{T} \mathbf{M}^{\prime} \tilde{\mathbf{X}} .
\end{aligned}
$$

The value of $\omega$ is real if $4 a c>=0$, and then $\omega^{2}$ will be real and greater than or equal to zero.

Without changing the eigenvalues, we can change the sign of the $P_{0}$ equations, giving

$$
\mathbf{K}^{\prime}=\left[\begin{array}{ccc}
\mathbf{K}_{S S} \mathbf{0} & \mathbf{K}_{P S}^{T} \\
\mathbf{0} & \mathbf{K}_{F F} & \mathbf{0} \\
-\mathbf{K}_{P S} & \mathbf{0} & -\mathbf{K}_{P P}
\end{array}\right], \quad \mathbf{M}^{\prime}=\left[\begin{array}{lll}
\mathbf{M}_{S S} \mathbf{0} & \mathbf{0} \\
\mathbf{0} & \mathbf{M}_{F F} \mathbf{0} \\
\mathbf{0} & \mathbf{0} & \mathbf{0}
\end{array}\right]
$$

Now

$$
\begin{aligned}
a=\tilde{\mathbf{X}}^{T} \mathbf{K}^{\prime} \overline{\mathbf{X}} & =\tilde{\mathbf{U}}^{T} \mathbf{K}_{S S} \tilde{\mathbf{U}}+\tilde{\mathbf{U}}^{T} \mathbf{K}_{P S}^{T} \overline{\mathbf{P}} \\
& +\overline{\boldsymbol{\phi}}^{T} \mathbf{K}_{F F} \tilde{\boldsymbol{\phi}}-\tilde{\mathbf{P}}^{T} \mathbf{K}_{P S} \tilde{\mathbf{U}}-\tilde{\mathbf{P}}^{T} \mathbf{K}_{P P} \tilde{\mathbf{P}}
\end{aligned}
$$

Recalling that $\mathbf{K}_{P P}$ is a diagonal matrix with all negative elements, we see that $a>=0$. Also,

$$
c=\tilde{\mathbf{X}}^{T} \mathbf{M}^{\prime} \tilde{\mathbf{X}}=\tilde{\mathbf{U}}^{T} \mathbf{M}_{s s} \tilde{\mathbf{U}}+\overline{\boldsymbol{\phi}}^{T} \mathbf{M}_{F F} \tilde{\boldsymbol{\phi}}
$$

and clearly $c>=0$.

This shows that $4 a c>=0$ so that $\omega^{2}$ is real and greater than or equal to zero.

\section{A.2. Number of sign changes in factorization}

Here we give the reason for the statement that for

$$
\mathbf{K}^{*}=\mathbf{L} \mathbf{D} \mathbf{L}^{T}=\mathbf{K}^{\prime}-\mu \mathbf{C}^{\prime}-\mu^{2} \mathbf{M}^{\prime},
$$

the number of eigenvalues below $\mu^{2}$ is equal to the number of negative elements in $D$ minus the number of $P_{0}$ variables. We explore this for a single $P_{0}$ variable in three steps (extension to multiple $P_{0}$ variables is straightforward).

(1) First we introduce the $\phi-U$ formulation, which has the Sturm sequence property. We replace the $P_{0}$ degree of freedom by a $\phi$ degree of freedom to obtain

$$
\begin{aligned}
& {\left[\begin{array}{lll}
\mathbf{K}_{S S S} & \mathbf{0} & \mathbf{0} \\
\mathbf{0} & \mathbf{K}_{F F} & \mathbf{K}_{A F}^{T} \\
\mathbf{0} & \mathbf{K}_{A F} & \mathbf{K}_{A A}
\end{array}\right]\left[\begin{array}{l}
\tilde{\mathbf{U}} \\
\hat{\boldsymbol{\phi}} \\
\hat{\phi}_{A}
\end{array}\right]-\omega\left[\begin{array}{lll}
\mathbf{0} & \mathbf{C}_{F S}^{T} & \mathbf{C}_{A S}^{T} \\
\mathbf{C}_{F S} & \mathbf{0} & \mathbf{0} \\
\mathbf{C}_{A S} & \mathbf{0} & \mathbf{0}
\end{array}\right]\left[\begin{array}{l}
\hat{\mathbf{U}} \\
\mathbf{K}^{\prime \prime} \\
\mathbf{C}^{\prime \prime}
\end{array}\right]} \\
& -\omega^{2}\left[\begin{array}{lll}
\mathbf{M}_{S S} & \mathbf{0} & \mathbf{0} \\
\mathbf{0} & \mathbf{M}_{F F} & \mathbf{M}_{A F}^{T} \\
\mathbf{0} & \mathbf{M}_{A F} & \mathbf{M}_{A A}
\end{array}\right]\left[\begin{array}{l}
\tilde{\mathbf{U}} \\
\hat{\mathbf{\phi}} \\
\mathbf{\phi}_{A}
\end{array}\right]=\left[\begin{array}{l}
\mathbf{0} \\
\mathbf{0} \\
0
\end{array}\right] \text {. }
\end{aligned}
$$

The system (35) has a single rigid body mode due to the fluid. By transforming to a standard eigenproblem, it can be shown that this system possesses the Sturm sequence property. 
As a consequence of the Sturm sequence property, the number of eigenvalues $\omega^{2}$ below $\mu^{2}$ in

$$
\mathbf{K}^{n *}=\mathbf{K}^{\prime \prime}-\mu \mathbf{C}^{\prime \prime}-\mu^{2} \mathbf{M}^{\prime \prime}=\mathbf{L}^{\prime \prime} \mathbf{D}^{\prime \prime} \mathbf{L}^{\mu T}
$$

is equal to the number of negative elements in $\mathbf{D}^{\prime \prime}$.

(2) In order to show that the $\phi-U$ formulation eigenvalues are the same as the $\phi-U-P_{0}$ eigenvalues (except for the zero eigenvalue), we will transform the system in eqn (35) to the $\phi-U-P_{0}$ formulation

We know that the system (35) has a zero eigenvalue

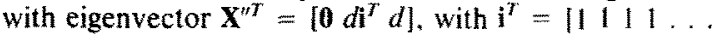
1] and $d$ is a constant. This means that

$$
\begin{aligned}
& \mathbf{K}_{F F} \mathbf{i}+\mathbf{K}_{A F}^{I}=\mathbf{0}, \\
& \mathbf{K}_{A F} \mathbf{i}+\mathbf{K}_{A A}-\mathbf{0} .
\end{aligned}
$$

We can write the eigenvector with eigenvalue $\omega^{2}$ as

$$
\left[\begin{array}{l}
\tilde{\mathbf{U}} \\
\hat{\boldsymbol{\phi}} \\
\hat{\phi}_{A}
\end{array}\right]=\left[\begin{array}{l}
\tilde{\mathbf{U}} \\
\tilde{\boldsymbol{\phi}}+\alpha \mathbf{i} \\
\alpha
\end{array}\right] \text {. }
$$

where $\alpha$ is a constant (which may be different from $d$ ). Substituting from eqn (39) into eqn (35), using eqn (37) and (38), and making the substitution $\tilde{P}=\rho_{F} \omega \alpha$, gives

$$
\begin{aligned}
& {\left[\begin{array}{lll}
\mathbf{K}_{s s} \mathbf{0} & \mathbf{A}^{T} \\
\mathbf{0} & \mathbf{K}_{F F} & \mathbf{0} \\
\mathbf{A} & \mathbf{0} & \mathbf{D}
\end{array}\right]\left[\begin{array}{l}
\tilde{\mathbf{U}} \\
\tilde{\phi} \\
\tilde{\mathbf{P}}
\end{array}\right]-\omega\left[\begin{array}{lll}
\mathbf{0} & \mathbf{C}_{F s}^{I} & 0 \\
\mathbf{C}_{F S} & \mathbf{0} & \mathbf{B}^{\prime} \\
\mathbf{0} & \mathbf{B} & \mathbf{0}
\end{array}\right]\left[\begin{array}{l}
\tilde{\mathbf{U}} \\
\tilde{\phi} \\
\tilde{\mathbf{P}}
\end{array}\right]} \\
& -\omega^{2}\left[\begin{array}{lll}
\mathbf{M}_{S S} & 0 & 0 \\
0 & \mathbf{M}_{F F} & 0 \\
0 & 0 & 0
\end{array}\right]\left[\begin{array}{l}
\tilde{\mathbf{U}} \\
\hat{\mathbf{\phi}} \\
\hat{\mathbf{P}}
\end{array}\right]=\left[\begin{array}{l}
0 \\
0 \\
0
\end{array}\right] \text {. }
\end{aligned}
$$

where

$$
\begin{aligned}
& \mathbf{A}=-\left(\mathbf{i}^{T} \mathbf{C}_{F S}+\mathbf{C}_{A S}\right) / \rho_{F}, \\
& \mathbf{B}=\left(\mathbf{i}^{T} \mathbf{M}_{F F}+\mathbf{M}_{A F}\right) / \rho_{F}, \\
& \mathbf{D}=-\left(\mathbf{i}^{T} \mathbf{M}_{F F} \mathbf{i}+\mathbf{i}^{T} \mathbf{M}_{A F}^{T}+\mathbf{M}_{A F} \mathbf{i}+\mathbf{M}_{A A}\right) / \rho_{F}^{2} .
\end{aligned}
$$

Evaluating the integrals for $\mathbf{A} . \mathbf{B}$, and $\mathbf{D}$ on an elemental basis shows that, with exact integration, $\mathbf{A}=\mathbf{K}_{P S}, \mathbf{D}=$ $\mathbf{K}_{P P}$, and $\mathbf{B}=\mathbf{C}_{P F}$ (so that we have recovered the $\phi-U$ $P_{0}$ formulation.) Therefore, every eigenvalue for $\phi-U$ (except the zero eigenvalue) is also an eigenvalue for $\phi-U$ $P_{0}$.

(3) Since the matrices for the $\phi-U$ formulation and the $\phi-U-P_{0}$ formulation are the same except for the last row and column, the first and higher associated constraint problems are the same (and form a Sturm sequence). This, with the fact that $\phi-U$ and $\phi-U-P_{0}$ have the same eigenvalues (except at zero). means that $D$ always has the same number of sign changes as $\mathrm{D}^{t}$. However. the $\phi-U-P_{0}$ formulation has no zero eigenvalue, so we have that the num. ber of sign changes in $\mathbf{D}$ is the number of eigenvalues below $\mu^{2}$ minus one.

\section{A.3. Orthogonality relation}

Our original eigenproblem is of the form

$$
\mathbf{K}^{\prime} \overline{\mathbf{X}}-\omega^{\prime} \overline{\mathbf{X}}-\omega^{2} \mathbf{M}^{\prime} \tilde{\mathbf{X}}=\mathbf{0} .
$$

By rewriting this eigenproblem in the standard form

$$
\mathbf{A} \mathbf{Y}=\omega \mathbf{B} \mathbf{Y} .
$$

where

$\mathbf{A}=\left[\begin{array}{ll}\mathbf{K}^{\prime} & \mathbf{0} \\ \mathbf{0} & \mathbf{M}^{\prime}\end{array}\right], \quad \mathbf{B}=\left[\begin{array}{ll}\mathbf{C}^{\prime} & \mathbf{M}^{\prime} \\ \mathbf{M}^{\prime} & \mathbf{0}\end{array}\right], \quad \mathbf{Y}-\left[\begin{array}{l}\dot{\mathbf{X}} \\ \omega \dot{\mathbf{X}}\end{array}\right]$,

we can use the orthogonality relation for the standard eigenproblem to derive an orthogonality relationship for our problem. Therefore.

$$
\mathbf{Y}_{i}^{T} \mathbf{B} \mathbf{Y},=0 ; \quad i \neq j
$$

or, multiplying out

$$
\overline{\mathbf{X}}_{i}^{T} \mathrm{C}^{\prime} \overline{\mathbf{X}}_{1}+\left(\omega_{i}+\omega_{\jmath}\right) \dot{\mathbf{X}}_{i}^{T} \mathbf{M}^{\prime} \dot{\mathbf{X}}_{\jmath}=0 ; \quad i \neq j .
$$

which is the orthogonality relation we use. 